\title{
¿Hablamos de lo mismo? Estudio contrastivo de programas de lenguas para fines específicos en dos universidades de Estados Unidos y España
}

\author{
Jesús García Laborda ${ }^{1}$ \\ Universidad de Alcalá de Henares
}

Luis G. Bejarano
Valdosta State University

RESUMEN

Las asignaturas de idiomas para fines específicos varían debido a su contexto y sus propósitos. Sin embargo, es posible encontrar rasgos comunes entre programas en dos países diferentes, con diferentes características y lenguas en cuestión. Es interesante entonces observar cómo funcionan diferentes asignaturas según sus rasgos específicos para destacar además de sus similitudes sus limitantes y retos. En este sentido, este trabajo aborda los casos de español para fines específicos en la Facultad de Lenguas Extranjeras de la Universidad Estatal de Valdosta en Estados Unidos y de inglés en la Facultad de Educación de la Universidad de Alcalá en España. El estudio contrastivo se refiere a las características de ambos programas, de sus asignaturas y alumnos, la metodología y los materiales utilizados para la instrucción, el uso de la lengua objetivo y la evaluación. El documento concluye que a pesar de la distinta naturaleza de los programas y asignaturas en cuestión se pueden encontrar más similitudes que diferencias de fondo, para que estudios futuros aborden estos contrastes en la elaboración de un marco común internacional.

Palabras clave: ESP, tecnología, diseño de asignatura, estudio de contraste, metodología, limitaciones

1 Corresponding author - Universidad de Alcalá, Departamento de Filología Moderna, Calle Trinidad, 3, 28802 Alcalá de Henares (Madrid), Spain.

Email: jesus.garcialaborda@uah.es 


\begin{abstract}
Language courses for specific purposes vary due to their context and purpose. However, it is possible to find common features between programs in two different countries, with different characteristics and languages in question. It is interesting then to observe how different courses work according to their specific characteristics to highlight in addition to their similarities their limitations and challenges. In this sense, this paper addresses the cases of Spanish for specific purposes in the Faculty of Foreign Languages of Valdosta State University in the United States and English in the Faculty of Education of the University of Alcalá in Spain. The contrastive study refers to the characteristics of both programs, their courses and students, the methodology and materials used for instruction, the use of the target language and evaluation. The paper concludes that in spite of the different nature of the programs and courses in question, more similarities can be found than differences of substance, so that future studies will address these contrasts in the elaboration of an international common framework.
\end{abstract}

Keywords: ESP, technology, course design, contrast study, methodology, limitations

\title{
1. Introducción
}

La historia de la enseñanza de lenguas para fines específicos probablemente se remonta a los tiempos de los griegos o de los romanos (Howatt, 1984). El aprendizaje de idiomas en aquella época se centraba en conocer las lenguas de sociedades "invasoras" en las nuevas colonias establecidas con fines fundamentalmente económicos y militares. Es decir, en no pocas ocasiones diplomáticos, generales y comerciantes establecían relaciones con pueblos locales que se basaban en el uso de traductores -mayormente locales- o en que los propios vencedores aprendiesen unas nociones básicas de las lenguas autóctonas. Hace más de 20 siglos el latín se establece como lingua franca (Ijsewijn, 2007), dominio que bajo las iglesias cristianas va a permanecer en Europa hasta la época de las colonizaciones ya en los siglos XVII y XVIII.

La llegada de España al nuevo mundo trae consigo la extensión del idioma español que llegará a tener una influencia poderosa en todo el continente americano (excepto en el norte de los Estados Unidos y Canadá, claramente dominados por Inglaterra y Francia). La independencia de los Estados Unidos conlleva la adopción del inglés como lengua administrativa de la nueva nación, tras la apretada decisión en perjuicio del alemán, votada en el congreso de Filadelfia de 1795 (Baron, nd). Aunque en este periodo ya aparecen los corpora de inglés legal, no se enfatiza su enseñanza internacionalmente por lo que no será hasta principios del siglo XX y especialmente después de la primera guerra mundial cuando se den auténticos indicios del desarrollo de las lenguas con fines profesionales o específicos 
(Hutchinson \& Waters, 1987, citado por García Laborda \& Litzler, 2015). En Estados Unidos, el español se ha convertido en su segunda lengua y en recurso invaluable de las relaciones culturales y comerciales dentro y fuera de sus fronteras geográficas. De hecho, con más de 50 millones, Estados Unidos se ha convertido en el segundo país del mundo con mayor número de hispano hablantes.

En este artículo nos centraremos en dos enfoques diferentes de la enseñanza de idiomas, ya sea del inglés en España o del español en Estados Unidos. Consideraremos entonces dos grupos disímiles, aunque ambos de pregrado, en la Universidad de Valdosta (VSU) en Georgia, Estados Unidos, que toman estos asignaturas de español $100 \%$ en línea y los estudiantes del Grado de Maestro de Primaria (de aquí en adelante, Magisterio) en inglés de la Universidad de Alcalá de Henares (UAH) en España que lo hacen de manera presencial. Después de evidenciar los rasgos distintivos, este trabajo analiza las similitudes inherentes a los programas en cuestión, a pesar de sus diferentes características y medios en que se enseñan. Los puntos básicos para este estudio contrastivo internacional se desprenden de las siguientes cuestiones:

¿Cuáles son los contrastes de los programas de idiomas para fines específicos, en dos países y con dos idiomas diferentes?

¿Qué características definen sus métodos y cuestionan sus limitaciones?

¿Qué puede aplicarse en ambos casos para su mejora y qué incluir al elaborar un marco común?

\section{Contextualización}

Las necesidades de los programas de lenguas para fines específicos están determinadas por diferentes contextos y enfoques, según veremos enseguida. En el marco en que nos movemos encontramos que, a pesar de su diferente naturaleza, los dos programas en cuestión comparten más de lo que aparentan. Los alumnos de VSU son anglohablantes que toman asignaturas de español con fines específicos para integrarlos a su campo profesional, mientras que los estudiantes españoles utilizarán el inglés en el aula e incluso en talleres o asignaturas pero no es la lengua en la que socialmente se desenvolverán. Además de estudiar español especializado para su futuro empleo, los alumnos de VSU lo aprenderán para la comunicación intercultural negociada de acuerdo con su carrera, mientras que los alumnos españoles se enfocarán más en las funciones directivas del lenguaje (DeCarlo, 1994, y también Bartanusz \& Sulova, 2003 en relaciones familiares), entendidas como aquellas funciones metalingüísticas que utilizan los maestros para dirigir y regular el 
funcionamiento de la clase. Por tanto, en el primer caso LSP se asocia con el aprendizaje de una segunda lengua (español) y en el otro con una lengua extranjera (inglés). Sin embargo, ambos casos comparten ciertos elementos que veremos más adelante, así como una filosofía similar en el diseño del programa curricular (figura 1).

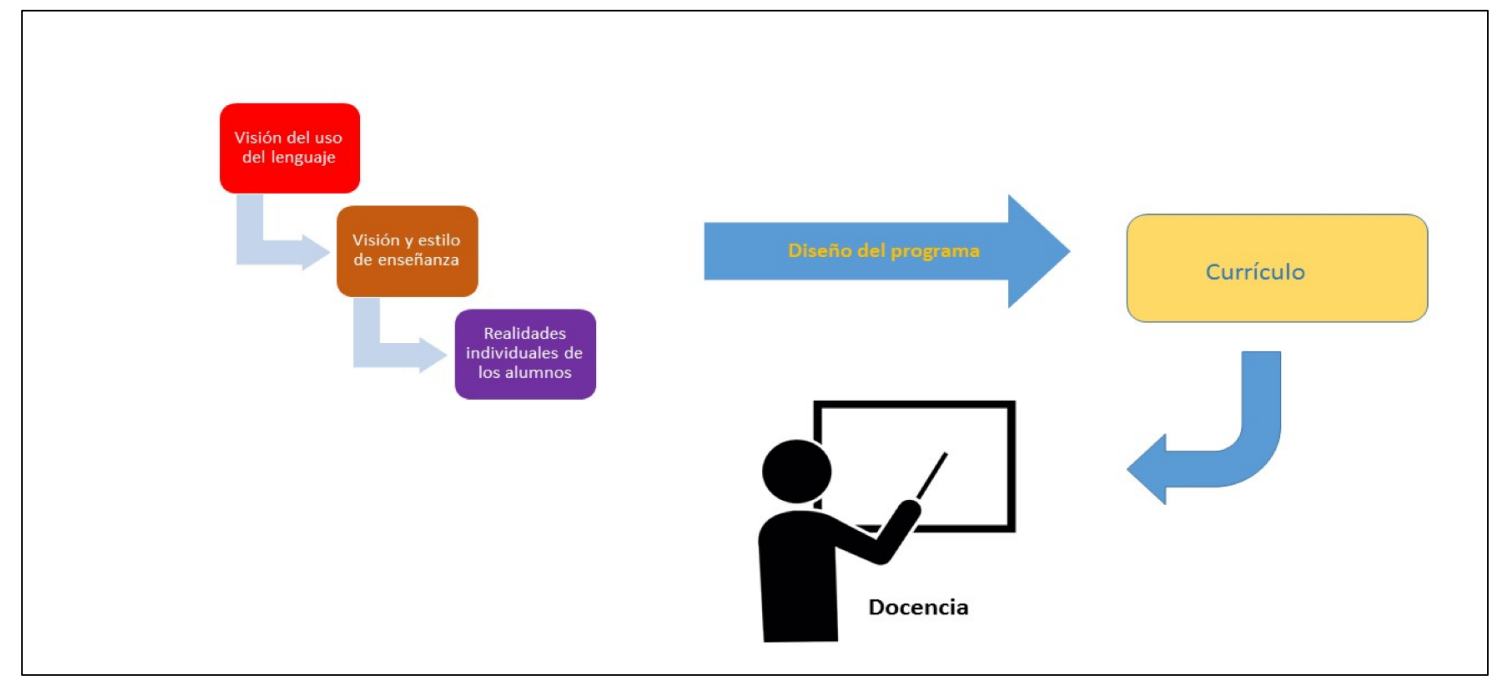

Figura 1: Diseño del currículo basado en una perfectiva de contenido y enseñanza

\section{Características de los alumnos en ambos programas}

Los alumnos de español de VSU son de tercero y cuarto año, de diversas titulaciones, quienes cursan asignaturas de español para fines específicos en línea. La primera asignatura para tercer año es conversación avanzada en español (Español 3012), lo complementa la asignatura sobre lengua y cultura (Español 3014), y para cuarto año la práctica de campo (Español 4500), y la investigación para profesionales (Español 4501). La universidad concede un certificado en español para las profesiones a aquellos alumnos que aprueben estas asignaturas en su área de especialización, sea ésta salud, negocios o criminalística. La mayoría de alumnos que toman estas asignaturas lo hace para cumplir su requisito de lengua extranjera aplicado a su carrera, o como una subespecialidad, aunque sin expectativas docentes.

Los alumnos de inglés de UAH, en la asignatura "Didáctica de la Lengua Inglesa" (430041-2016-17) por su parte, están estudiando su cuarto y última asignatura de la titulación de Magisterio y sus clases están orientadas al aprendizaje de conceptos, 
ideas y vocabulario específico cuya aplicación se dará en el aula de enseñanza primaria durante su carrera profesional. En lo referente a su uso profesional, los conocimientos, sobre todo, se centran en la adquisición en lengua extranjera de dos tipos de registros: metalenguaje y lenguaje asociado a procesos cognitivos y didácticos. Mientras que el primero podrían haberlo comenzado a conocer los alumnos a través de su experiencia como discentes, el segundo sólo se puede adquirir en el aula universitaria. Asimismo, tienen dos asignaturas que les sirven de base para la adquisición de conocimientos profesionales: 1) aprendizaje integrado de contenidos y lengua inglesa (AICLE/CLIL) (430040) y 2) Didáctica de la lengua inglesa (430041) que es en la que nos centramos en este artículo como dijimos más arriba.

\section{Descripción de la situación de las asignaturas en los dos programas}

La asignatura de conversación aplicada de español para profesionales de VSU, (Español 3012), consiste de la práctica del discurso formal e informal para la comunicación en contextos profesionales tales como administración de negocios y finanzas, criminalística y áreas de la salud, como la enfermería, y se centra en refinar la fluidez expresiva a través de presentaciones y experiencias prácticas. La asignatura de cultura y conversación avanzada (Español 3014), consiste de la práctica avanzada de los mismos contextos profesionales. La práctica de conversación se centra en discutir y debatir cómo las realidades culturales, históricas y contemporáneas del mundo de habla hispana afectan el lenguaje y la comunicación oral. Por su parte, la asignatura de practicum relacionada con las profesiones (Español 4500), consiste de una práctica supervisada en una comunidad hispano hablante o durante el viaje a un país hispano en el verano. Los alumnos interesados en enfermería pueden, por ejemplo, realizar su práctica trabajando como asistentes en instituciones como la Cruz Roja o en centros de salud locales en la ciudad hispana donde hacen su asignatura de verano. Del mismo modo, los alumnos de criminalística pueden realizar prácticas en cortes judiciales, estaciones de policía o prisiones locales. La asignatura de proyecto de investigación para profesionales (Español 4501), consiste del uso práctico y profesional del español oral y escrito hacia el desarrollo de un proyecto comunal para mejorar la comunicación con clientes hispano hablantes.

Los estudiantes españoles, por su parte, realizan su aprendizaje de lenguas para fines específicos como una parte significativa de dos asignaturas: Content Language Integrated Learning (CLIL) y Didáctica de la Lengua Inglesa. Mientras que la asignatura de CLIL se centra en cómo comunicar contenidos curriculares que no sean de la asignatura de inglés en dicha lengua, la de Didáctica se centra en la metodología y la instrucción en la asignatura de Inglés. A través de las dos 
asignaturas, los estudiantes españoles, por tanto, adquieren el léxico relacionado con su docencia específicamente inmerso en los contenidos de una y otra manera. Es decir, estos futuros profesores de inglés deberán usar o incluso haber usado este lenguaje en sus prácticas docentes o en clases no reguladas. Las enseñanzas de estas dos asignaturas son fundamentales para alumnos que ya han hecho prácticas docentes ya que sirven para fundamentar sus observaciones realizadas en esos periodos de prácticas y, en muchos casos, como profesores de inglés en actividades extraescolares, en academias o como tutores individuales. En estas asignaturas adquieren el lenguaje necesario para poder realizar su labor docente.

\section{Metodología y materiales de ambos programas}

Los asignaturas en VSU ya descritos buscan alejarse del encasillamiento tradicional enfocado en traducción y memorización de frases básicas para situaciones con módulos muy determinados. Más bien se quiere que el alumno de nivel intermedio refine su fluidez a través de discusiones y experiencias prácticas que reflejen contextos auténticos del mundo hispano, incluyendo los Estados Unidos. Cada área de especialización tiene un libro de texto especializado, y una tabla de contenidos, así como la lista de temas que le ayuda al alumno a decidir qué área elegir en caso de indecisión. Estas asignaturas buscan adaptarse a diferentes necesidades y estilos de aprendizaje a través de la instrucción en línea usando herramientas multimedia síncronas y asíncronas como Wimba, Blackboard Collaborate y aulas virtuales de videoconferencia similares, además de grabaciones de video y entornos sociales en línea como YouTube y de realidad virtual como Second Life. Estos recursos permiten flexibilidad en la programación para los estudiantes. Las reuniones en línea sincrónicas requeridas individualmente y el trabajo en grupo brindan a los alumnos un mejor manejo del tiempo al interactuar con el instructor. Los alumnos necesitan aprender cómo utilizar las tecnologías incorporadas en las asignaturas más allá de los medios sociales virtuales. Éstos también ofrecen tutoriales y soporte técnico, ya sea directamente con las empresas diseñadoras de las plataformas o la oficina de soporte tecnológico dispuesta por la universidad.

Los alumnos de la UAH asimismo se han visto en un profundo proceso de modernización de su discencia a través del uso de plataformas digitales y la incorporación de tecnologías (especialmente Web 2.0) en su asignatura. Igualmente, estos discentes trabajan fundamentalmente de manera colectiva. Por ejemplo, crean blogs, videos educativos y desarrollan páginas web sociales profesionales como Linkedin. En clase suelen recibir los contenidos a través de clases magistrales de 20 a 40 minutos seguidas de ejercicios de reflexión y en algunas ocasiones del estudio de casos por grupos. Éstos pueden llevar un seguimiento de manera individual. Generalmente el estudio se guía a través de la plataforma virtual que 
sirve para proveer a los alumnos con la información necesaria para su propio estudio autónomo y la ampliación de contenidos de aquellos recibidos en el aula. Más infrecuentemente pueden recibir tareas escritas que siempre se elaboran fuera del aula, como en el caso de la creación de blogs. También se ha incrementado su competencia oral mediante la reducción del número de alumnos en el aula. Esto se complementa con la actividad en los periodos de prácticas que los introducen de manera real en el trabajo y responsabilidades del aula y les obligan a trabajar en inglés desde las primeras asignaturas de su carrera. Ésta es la comparación que se establece de los intereses metodológicos generales en ambos programas:

\begin{tabular}{|c|c|c|c|c|c|c|c|c|}
\hline & \multicolumn{4}{|c|}{ Actividades grupales } & \multicolumn{4}{|c|}{ Actividades individuales } \\
\hline & $\begin{array}{l}\text { Resolución de } \\
\text { casos }\end{array}$ & $\begin{array}{l}\text { Proyectos } \\
\text { colaborativos TIC }\end{array}$ & Debates & $\begin{array}{l}\text { Role- } \\
\text { plays }\end{array}$ & $\begin{array}{l}\text { Lecturas } \\
\text { individuales }\end{array}$ & $\begin{array}{l}\text { Escritura: } \\
\text { ensayos, } \\
\text { informes, } \\
\text { otros }\end{array}$ & $\begin{array}{l}\text { Audición: } \\
\text { clase, } \\
\text { podcasts, } \\
\text { You tube }\end{array}$ & Otros \\
\hline UAH & Y & $x$ & $x$ & $x$ & $x$ & $\mathrm{Y}$ & $\mathrm{Y}$ & Y \\
\hline VSU & Y & & & $x$ & $\mathrm{Y}$ & Y & Y & \\
\hline
\end{tabular}

El enfoque inductivo de las asignaturas de la $\mathrm{UAH}$, por tanto, se basa primero en la presentación del lenguaje que utilizarán después para las simulaciones y resolución de problemas y situaciones. Si bien es cierto que su conocimiento de inglés general les dará cierta seguridad, es indudable que la resolución de las tareas en clase requiere el dominio de fórmulas y elementos lingüísticos que han sido previamente introducidos por el instructor. En este sentido, la alfabetización digital y dentro de la misma el vocabulario específico adquiere todo su potencial y añade seguridad en la respuesta a las tareas. En general, los alumnos de la UAH utilizan materiales ya creados específicos para docentes que son muy comunes en internet (García Laborda, 2013) tales como juegos, crucigramas, ejercicios auto corregibles de lectura, gramática o comprensión auditiva (Podcasts), etc. Sin embargo, se enfatiza el uso de materiales de aprendizaje autónomo.

El aprendizaje autónomo y colaborativo que aquí mencionamos tiene una importancia vital en las asignaturas que presentamos (García Laborda, Bejarano \& Simons, 2012). Así, la tecnología tiene un papel destacado en los dos tipos de aprendizaje. La tecnología, facilita el aprendizaje tradicional en forma de input generalmente por el uso de internet tradicional (Web 1.0), por un lado y permite la comunicación dirigida y social (Web 2.0), por otro. Por eso, la guía de cómo usar las herramientas tecnológicas es importante de cara a la orientación, actuación y desarrollo de los alumnos y en ambos casos se asocia a las necesidades del currículo, los objetivos de la asignatura y las actividades generadas para atender a las necesidades receptivas y directivas de los alumnos (García Laborda \& Litzler, en 
prensa). García Laborda (2011) establece una clasificación de los distintos tipos de herramientas informáticas para la enseñanza de lenguas para fines específicos en los cuales se basan ambos autores en la selección de herramientas y materiales de aprendizaje. Para el caso que nos ocupa podemos resumir que las herramientas utilizadas por los alumnos en UAH y VSU son las siguientes:

\begin{tabular}{|c|c|c|c|c|c|c|c|c|c|}
\hline & \multicolumn{4}{|c|}{ En el aula o virtualmente } & \multicolumn{2}{|c|}{ Ambos } & \multicolumn{3}{|c|}{ Aprendizaje autónomo } \\
\hline & $\begin{array}{l}\text { Libro } \\
\text { de } \\
\text { texto }\end{array}$ & Internet & $\begin{array}{l}\text { Fichas } \\
\text { creadas por } \\
\text { el profesor }\end{array}$ & $\begin{array}{l}\text { Materiales } \\
\text { reales del } \\
\text { aula de } \\
\text { primaria }\end{array}$ & $\begin{array}{l}\text { Plataforma } \\
\text { de } \\
\text { aprendizaje }\end{array}$ & $\begin{array}{l}\text { Diccionarios y } \\
\text { referencia }\end{array}$ & $\begin{array}{l}\text { Media } \\
\text { Social } \\
\text { (blog, } \\
\text { Linkedin, } \\
\text { You } \\
\text { Tube) }\end{array}$ & Podcast & $\begin{array}{l}\text { Otros } \\
\text { elementos } \\
\text { de } \\
\text { aprendizaje } \\
\text { móvil }\end{array}$ \\
\hline UAH & & $x$ & $\mathrm{Y}$ & $x$ & & $x$ & $x$ & $\mathrm{Y}$ & Y \\
\hline VSU & $X$ & $x$ & & & & $x$ & $\mathrm{Y}$ & & $\mathrm{Y}$ \\
\hline
\end{tabular}

En el caso del programa de la UAH, los alumnos utilizan un compendio de las herramientas que se ilustran a continuación, integrándolas a través de la creación de un blog (figura 2) que incluye 2 videos de Vímeo/YouTube de creación propia y Linkedin, además de búsquedas en línea sobre un tema central y una selección de recursos en línea para la docencia de inglés en la enseñanza primaria (García Laborda \& Litzler, en prensa). El siguiente diagrama ilustra el tipo de herramientas virtuales que son comúnmente usadas en las asignaturas de los programas aquí referidos, sean en línea, híbridos o tradicionales (figura 2):

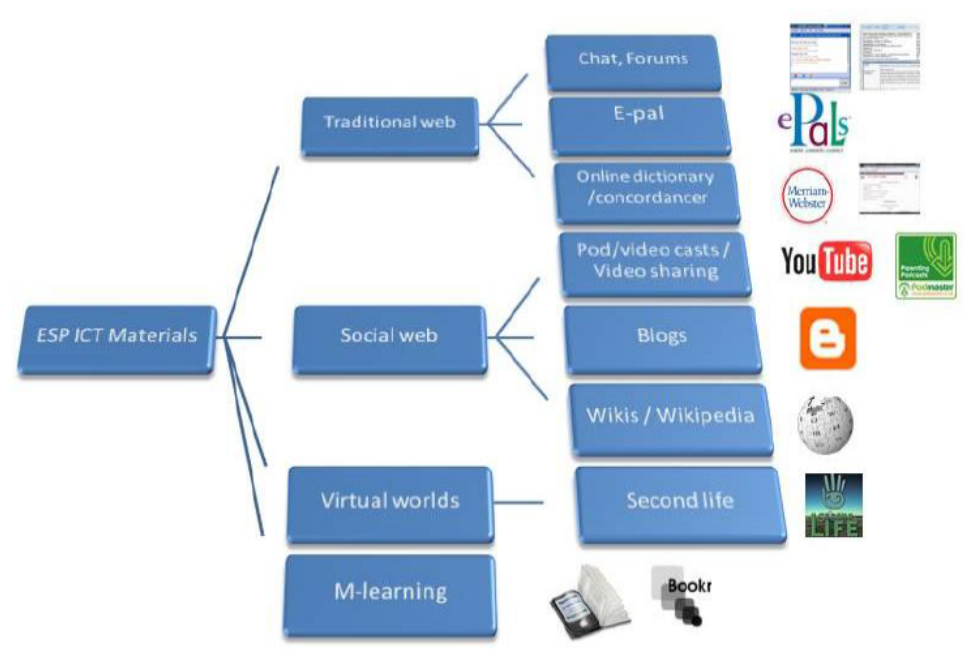

Figura 2. Clasificación de elementos web 2.0 para la enseñanza de lenguas para fines específicos (García Laborda, 2011)

Como hemos dicho, el desarrollo de un blog es una tarea obligatoria grupal que refleja el uso de la lengua (ver el siguiente apartado) y también la capacidad de los 
alumnos al buscar fuentes y actividades en internet. De esta manera tienen la oportunidad de desarrollar sus destrezas explorando determinadas herramientas como el vídeo del sitio Web (http://clilvialerosdidactics.weebly.com/videos.html) (figura 3).

Como se ve en la figura 3 , el blog sirve como elemento social ya que informa y permite el trabajo colaborativo. Asimismo, incluye el acceso a otras páginas y facilita la escritura. Por ejemplo, aquí se ve como los alumnos desarrollan varias destrezas como la escritura y el habla (http://clilvialerosdidactics.weebly.com/videos.html, operativo 18 de marzo de 2017). Igualmente, utilizan distintos géneros como la exposición, la dramatización, el informe, etc. de los que se hablará en la sección siguiente. También se fomenta el desarrollo de la capacidad profesional mediante la creación de sus propios sitios en Linkedin.

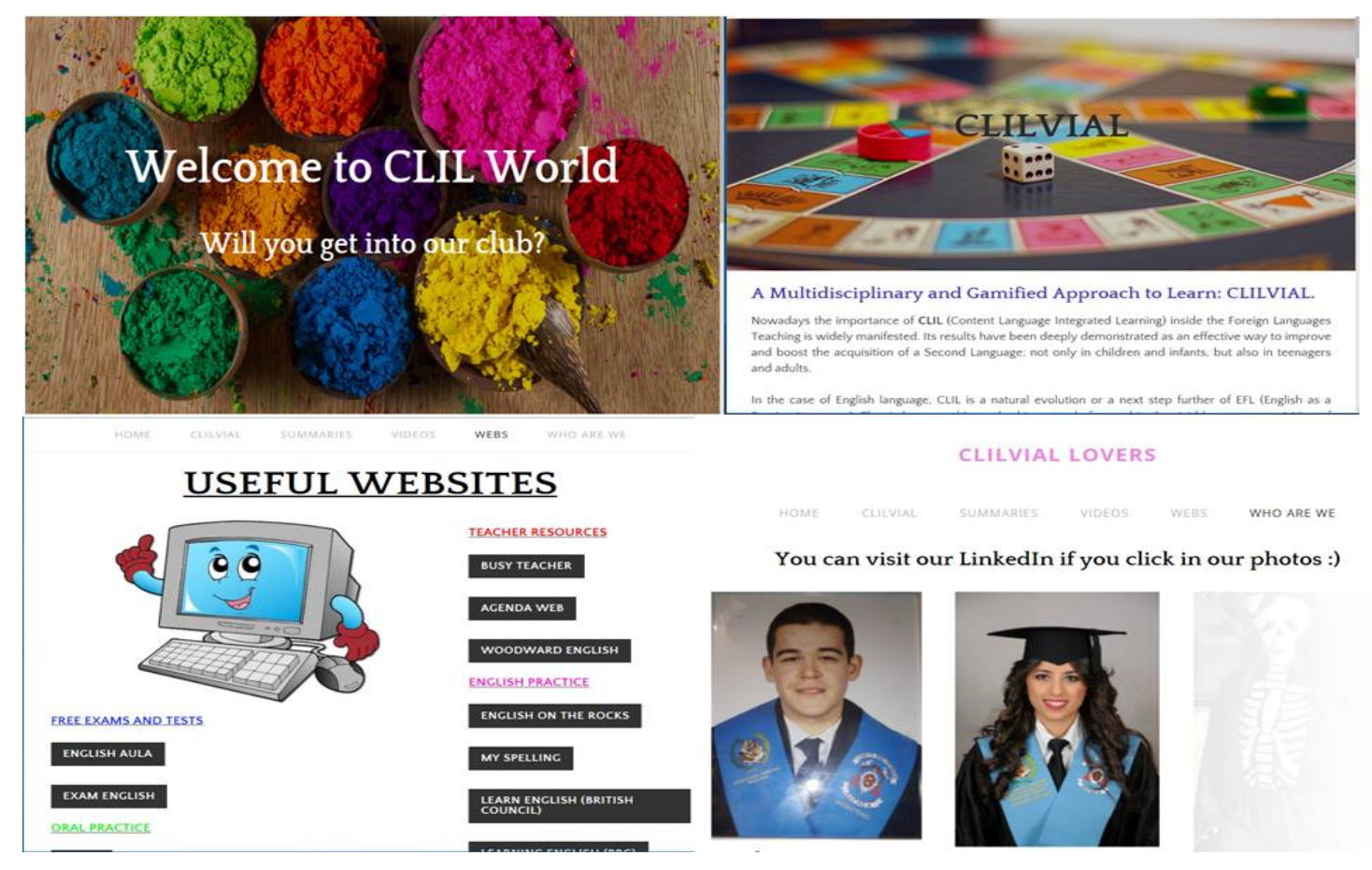

Figura 3. Distintas interfaces del blog Clilvial creado en la asignatura de Didáctica del Inglés (UAH).

\section{Uso de la lengua}

Una de las cuestiones centrales a la enseñanza de lenguas para fines específicos se refiere al uso del español en el programa de VSU y del inglés en el de UAH. Es evidente que dicho uso debe desarrollarse en torno a las futuras necesidades de las 
especialidades. Este aspecto queda claro desde la propia descripción de los dos grupos y de sus asignaturas específicas. El siguiente cuadro muestra las áreas a las que se presta más atención en cada caso:

\begin{tabular}{|c|c|c|c|c|c|c|c|c|}
\hline & \multicolumn{4}{|c|}{ Uso de la lengua } & \multicolumn{4}{|c|}{ Destrezas } \\
\hline & $\begin{array}{c}\text { Organización } \\
\text { discursiva y } \\
\text { sintaxis }\end{array}$ & Gramática & Vocabulario & Pronunciación & Lectura & Escritura & Audición & Habla \\
\hline UAH & $x$ & Y & $x$ & Y & $x$ & Y & & $x$ \\
\hline VSU & & $\mathrm{Y}$ & $x$ & Y & $x$ & $\mathrm{Y}$ & $X$ & $X$ \\
\hline
\end{tabular}

Tabla 3: Comparación de uso y destrezas de la lengua entre UAH y VSU

Los alumnos en las asignaturas de VSU disponen de abundantes oportunidades para desarrollar el aprendizaje de las cuatro destrezas de la lengua. Los delineamientos de las asignaturas de lengua extranjera siguen los estándares dispuestos por ACTFL (consejo norteamericano para la enseñanza de lenguas extranjeras, https://www.actfl.org/publications/guidelines-and-manuals/actflproficiency-guidelines-2012) para el nivel intermedio medio e intermedio alto, enfatizando más la escucha y el habla. Sin embargo, por la naturaleza de estas asignaturas en línea es difícil para el instructor hacer un seguimiento continuo del desarrollo de destrezas del grupo, y se recurre más a enfocarse en el nivel individual. El uso del español como lengua objetivo implica necesariamente desarrollar herramientas de comunicación más allá de las opciones de la plataforma instruccional de programas en línea Blazeview para realizar interacciones individuales y grupales. Así que además de las discusiones, correo, mensajes de chat, y anuncios, el instructor debe implementar herramientas adicionales para las actividades orales como entrevistas individuales y conversaciones en grupos usando Blackboard Collaborate, Wimba, Skype, etc. Para ayudar a la motivación de los estudiantes en línea existen diferentes herramientas interactivas que se integran en estas asignaturas, entre otras, la función de reconocimiento de voz para mejorar la pronunciación que ofrecen Transparent Language y Tell me More.

Una actividad que ilustra bien la integración tanto de las destrezas comunicativas como del contenido lingüístico de las asignaturas con los recursos tecnológicos disponibles es la creación de módulos de conversación. Por ejemplo, los alumnos interesados en español para negocios reciben como asignación inventar dos escenas a partir de una situación general que normalmente es: "Ustedes son estudiantes de intercambio en Panamá, han perdido su dinero y necesitan conseguir un trabajo, finalmente deben abrir una cuenta de ahorros". Los alumnos en pares deben usar la red, leyendo páginas en español, para ubicar en Ciudad de Panamá una agencia de empleos, conseguir información auténtica del sitio e inventar una entrevista con el gerente encargado. Seguidamente, deben explorar 
virtualmente el banco local que ofrezca el más alto interés en las cuentas de ahorros y simular una entrevista con el encargado de las cuentas para abrir el tipo de cuenta que más convenga. Al escribir los diálogos de estas dos sencillas escenas los alumnos tienen la oportunidad de utilizar el vocabulario y funciones relativas al empleo y los servicios bancarios, además de explorar información auténtica directamente escuchando y leyendo en español. Los alumnos deben finalmente preparar una presentación en Power Point que puede incluir fotos y videos cortos de YouTube, y deben actuar (role-play), los diálogos de las escenas escritas en video conferencia con el instructor. Los alumnos son evaluados de acuerdo con una rúbrica para esta actividad, que incluye exploración y lectura de páginas web en español, ilustración de la presentación y desempeño oral. Una opción adicional para desarrollar destrezas orales es el uso de las plataformas comerciales en línea mediante las cuales los alumnos pueden interactuar en tiempo real con hablantes nativos calificados en países hispano hablantes.

También en la UAH se trabajan las cuatro destrezas a un nivel avanzado (B2.2 o C1 en Marco Europeo para Referencia de las Lenguas O CEFR, http://www.cambridgeenglish.org/Images/126011-using-cefr-principles-of-good-

practice.pdf), el énfasis se pone en aquellas actividades que van a ser más útiles y necesarias para los futuros maestros tales como presentaciones orales, role-plays, monólogos o expresión de forma de trabajo. Por tanto, se valora la interacción y el uso de determinadas formas lingüísticas y vocabulario más comunes entre los docentes. Una parte considerable del tiempo se dedica a desarrollar determinados usos del lenguaje oral que serán comunes en su actividad profesional, entre los que se incluyen los siguientes:

Expresión de la información mediante mini-presentaciones.

Solicitud de interacción con los alumnos a través de role-plays de minilecciones (García Laborda \& García Esteban, 2016).

(4) Demanda de genuina información en la interacción con pares y profesor. Lenguaje no verbal a través de demostraciones.

Mucho de este trabajo se hace en pequeñas cápsulas dentro de cada clase con el fin de promover la interacción teoría-práctica. A esto se añaden numerosos ejemplos extraídos de las páginas web de universidades de todo el mundo y de YouTube. En lo referente a la escritura, una destreza que quizás no se practica tanto como en 
otras áreas de ESP, se sigue un enfoque textual ya que para los profesores la escritura responde a la necesidad de comunicarse primariamente (Hyland, 2004; Johns, 2002). Por ello, se trabajan los blogs con entradas más o menos largas con el fin de desarrollar la competencia comunicativa, pero al tratarse de escritura muchas veces colectiva, la interacción se hace fundamental. De esta manera, entra a formar parte un aprendizaje socio-constructivista, porque la escritura se ve mediada por el desarrollo de escritos formales para padres, alumnos, administradores o instituciones con sus convenciones privativas (García Laborda \& García Esteban, 2016).

En lo referente a la lectura también encontramos unos géneros similares a la escritura ya que los alumnos tienen que leer artículos (dos), en alguna ocasión crear pequeñas cartas, por supuesto redacciones de temas específicos y en su proceso de escritura colectiva también leen (y escuchan) el comentario o feedback producido por los compañeros. Recientemente se ha observado la posibilidad de añadir cartas o e-mails, informes sobre alumnos, etc. pero esta asignatura no es tan amplia. De esta manera, podemos observar entonces que prevalecen las interacciones localizadas entre los distintos agentes del aprendizaje profesor-alumno-alumno (Barton \& Hamilton, 1998).

Observamos entonces que ambos programas ponen atención al desarrollo de las cuatro destrezas comunicativas, aunque el énfasis varíe ligeramente dependiendo de la asignatura específica y del tema y contenidos alrededor del vocabulario en cuestión. En ambos programas no se hace mucho énfasis en la precisión gramatical o escrita, para favorecer más la lectura y el habla. La expresión oral y la corrección de la pronunciación se hace difícil en ambos programas, aunque por la naturaleza individualizada de los asignaturas en línea los alumnos no sienten la intimidación para hablar que sí pueden sufrir en un formato presencial. Probablemente no haya un área donde el eclecticismo tenga más cabida que en la enseñanza de lenguas para fines específicos debido a la necesidad de individualizar la enseñanza (Long, 2005). En gran parte esta consideración tiene su raíz y justificación en el diseño del currículum, en el número de estudiantes, en el contexto profesional, en los resultados y aplicaciones finales, etc. (García Laborda \& Litzler, 2015).

\section{Evaluación}

La evaluación de los alumnos es parte del proceso de aprendizaje y busca establecer hasta qué punto se cumplen los objetivos trazados en el programa de estudio. Los estudiantes de las asignaturas de español en línea para fines específicos de VSU son evaluados de una manera más compleja que la evaluación en una asignatura presencial. Por tanto, se requiere integrar al componente humano y pedagógico el 
tecnológico, que incluye los recursos y materiales que faciliten no sólo la comunicación del alumno con el contenido sino con su instructor. La plataforma instruccional para asignaturas en línea Blazeview brinda al instructor opciones de evaluación básicas como tareas, exámenes, rúbricas, notas y evaluación por pares. Sin embargo, el instructor debe contar con recursos adicionales para complementar la evaluación como son la supervisión externa de la toma de exámenes individuales con pautas muy específicas basadas en la confiabilidad. Contactar la entidad supervisora de las pruebas escritas a distancia demanda tiempo y esfuerzo adicional por parte del instructor, lo que normalmente tanto institución como alumnos ignoran.

El programa de las asignaturas de VSU establece tres instancias principales para evaluación con un componente oral, una prueba de nivelación considerada también como un diagnóstico inicial, una prueba de medio semestre y una evaluación final, que normalmente incluye una presentación en Power Point. Se dispone normalmente de rúbricas para evaluaciones orales, entrevistas y presentaciones, así como de guías de estudio. De esta forma y mediante el uso de los recursos ya referidos se busca establecer el aprovechamiento de cada alumno según las diferentes tareas para determinar si corresponde a las expectativas de la asignatura y si no, cómo ayudarle. La frecuencia de interacción del instructor con sus alumnos ya sea individualmente o en grupos y el medio utilizado son determinantes para el aprovechamiento de la asignatura y para una percepción positiva por parte de los estudiantes. Del mismo modo, la disponibilidad continua del instructor y su función de facilitador son esenciales para que el alumno se sienta apoyado en su proceso de aprendizaje durante todo el cuatrimestre. La importancia de la evaluación en estas asignaturas obliga a una planeación y dedicación diferente a la tradicional en el aula de clase. Este tipo de asignaturas de lengua en línea implica una participación mayor del instructor, ya que como decíamos anteriormente, debe asumir en muchos casos el rol de tutor individual. Así, el proceso enseñanza-aprendizaje puede llegar a individualizarse hasta cierto punto de acuerdo con las exigencias de la asignatura. Es siempre importante además de valorar los comentarios de los alumnos al evaluar su aprovechamiento, incluir los componentes de la evaluación en los estándares de futuros programas.

La evaluación en la Universidad de Alcalá es continua y en ella participan tres factores fundamentales: dos exámenes, la evaluación del blog y la observación de los agentes educativos (autoevaluación, evaluación de pares y observaciones del profesor). Los exámenes incluyen sobre todo las secciones teóricas mientras que el blog ha sido ampliamente explicado anteriormente y se puede encontrar una descripción más detallada en García Laborda \& Litzler (2017). Finalmente, se solicita a los alumnos que autoevalúen su trabajo en toda la asignatura holísticamente y las 
presentaciones orales grupales son evaluadas tanto por el profesor como por los propios compañeros. La plataforma informática de la universidad conocida como campus virtual se usa fundamentalmente para el uso y seguimiento de las partes informales de la evaluación y la orientación de la asignatura. Consideramos entonces que una de las fortalezas de la evaluación en la asignatura es ir más allá del tradicional uso de la lengua y ser capaces de conocer los distintos géneros que afectan al área de especialización y el desarrollo profesional del individuo (por ejemplo, en el desarrollo de Linkedin, como se dijo anteriormente). De esta manera, se le da un realismo a la evaluación que raramente se obtiene en ambientes académicos y que consideramos fundamental para la competencia profesional y empleabilidad de los futuros maestros. Esto además permite evaluar tanto el conocimiento de la lengua como ciertas capacidades profesionales que deben comenzar a desarrollarse en la universidad. La tabla 4 pone en perspectiva el contraste de los programas de VSU y UAH con respecto a la evaluación basada en procesos y pruebas predominantemente orales.

\begin{tabular}{|c|c|c|c|c|c|c|c|c|}
\hline & \multicolumn{4}{|c|}{ Procesos } & \multicolumn{4}{|c|}{ Pruebas } \\
\hline & $\begin{array}{c}\text { Rúbrica } \\
\text { estandarizada }\end{array}$ & $\begin{array}{l}\text { Supervisión } \\
\text { externa }\end{array}$ & $\begin{array}{l}\text { Evaluación } \\
\text { externa }\end{array}$ & $\begin{array}{l}\text { Prueba } \\
\text { de } \\
\text { nivelación }\end{array}$ & $\begin{array}{c}\text { Examenes } \\
\text { mitad y } \\
\text { Final de } \\
\text { asignatura }\end{array}$ & $\begin{array}{c}\text { Blogy } \\
\text { aplicaciones } \\
\text { informáticas }\end{array}$ & $\begin{array}{l}\text { Entrevista oral, } \\
\text { presentaciones, } \\
\text { role play }\end{array}$ & $\begin{array}{l}\text { Observación } \\
\text { del profesor }\end{array}$ \\
\hline UAH & X(IATEFL) & & & $x$ & $\mathrm{X}$ & $Y$ & & $x$ \\
\hline VSU & X(CEFR) & $Y$ & $X$ & $x$ & $x$ & $x$ & $X$ & $x$ \\
\hline
\end{tabular}

Tabla 4: Comparación de actividades grupales e individuales entre UAH y VSU

\section{Otros rasgos diferenciadores entre las asignaturas de VSU y UAH}

En respuesta a las preguntas planteadas en la introducción, conviene decir que aunque ambos programas presentan diferencias desde las perspectivas de los objetivos y justificación de los estudiantes, presentan similitudes claramente significativas.

Los dos programas tienen claras similitudes en referencia al trabajo autónomo fuera y dentro del aula. Primero, hay un claro interés por el lenguaje específico que, generalmente, se encuentra también en corpus especializados. Por tanto, se desarrollan más algunos registros que otros (Flowerdew \& Peacock, 2001) dejándose generalmente atrás la corrección gramatical y promoviendo los usos específicos de la materia basados en las necesidades del alumno (Corder, 1973), y en el uso de las funciones lingüísticas. En ambos casos, el currículo viene impuesto por la especialización, lo que de alguna manera contrasta con la idea de Hutchinson \& Waters (1987) de que es precisamente la conjunción de la visión de aprendizaje y 
la de la lengua, las que son factores directores en la creación de la asignatura.

Según se ha presentado, los contextos también son diferentes y seguramente inciden en la motivación de los alumnos. Mientras los alumnos de la UAH tienen una asignatura más académica, el hecho de que los alumnos de VSU se preparen a ejercer una determinada profesión no limita necesariamente su interés en alcanzar éxito en su estudio de la lengua específica que, además, es importante para establecer unas relaciones directas y fluidas con los agentes que trabajan (ya sean pacientes, otros colegas, administradores, etc.). Asimismo, la oportunidad de desarrollar actividades exploratorias como la anteriormente descrita de VSU le brinda al alumno el conocimiento integrado de componentes lingüísticos y culturales aplicados un área específica, como la de los negocios. De este modo, se logra integrar el beneficio de la tecnología al aumento de las posibilidades de éxito en el aprendizaje de la lengua objetivo, enfatizando las destrezas orales.

Como se ha expuesto, las asignaturas analizadas gozan de más similitudes que de diferencias. Especialmente el uso más avanzado de recursos de videoconferencia en el caso de VSU, es algo que puede ser aplicado al trabajo autónomo de los alumnos de UAH. También conviene enfatizar metodologías que mejoren la expresión y, en lo posible, incrementen los conocimientos discursivos $y$, sobre todo, patrones interculturales. Asimismo, una continua revisión de las necesidades de los alumnos sería más que deseable.

Si hasta ahora se vieron una serie de rasgos comunes, en este momento trataremos de mostrar unos factores limitantes entendidos como aquellos aspectos que indican problemas que deberían solucionarse. Por ello, elegimos una perspectiva más esquemática con el fin de que se aprecien esos rasgos distintivos.

\begin{tabular}{lll}
\hline \multicolumn{1}{c}{ Factor } & Valdosta State & Universidad de Alcalá \\
\hline $\begin{array}{l}\text { Interacción } \\
\text { profesor- } \\
\text { Alumno }\end{array}$ & $\begin{array}{l}\text { La relación se retrasa ligeramente y no } \\
\text { tienen retroalimentación inmediata. }\end{array}$ & $\begin{array}{l}\text { Al ser presencial, en principio es } \\
\text { fluida y negociada. Los alumnos } \\
\text { negocian significados con el } \\
\text { profesor. }\end{array}$ \\
\hline $\begin{array}{ll}\text { Competencia } \\
\text { meta }\end{array}$ & El aprendizaje se limita a situaciones & $\begin{array}{l}\text { El aprendizaje es mucho más } \\
\text { amplio y conlleva un desarrollo } \\
\text { comunicativas cotidianas que no se }\end{array}$ \\
& aplican a estudios ulteriores (como el ámbito práctico (el \\
& lecturas o comunicaciones profesionales & aula misma) como la \\
& entre colegas). & $\begin{array}{l}\text { adquisición lingüística para la } \\
\text { comprensión de manuales y } \\
\text { conferencias. Por tanto, es más } \\
\text { holística que la de VSU. }\end{array}$ \\
& & La presencialidad tiene un \\
\hline Limitaciones & A pesar de la facilitación en la & \\
\hline
\end{tabular}




\begin{tabular}{|c|c|c|}
\hline $\begin{array}{l}\text { en la } \\
\text { comunicación } \\
\text { profesor- } \\
\text { alumno }\end{array}$ & $\begin{array}{l}\text { comunicación, los alumnos no siempre } \\
\text { responden a tiempo a los anuncios del } \\
\text { instructor y con frecuencia no cumplen } \\
\text { los plazos fijados para entregar una } \\
\text { asignación. }\end{array}$ & $\begin{array}{l}\text { factor activo en el cumplimiento } \\
\text { de fecha y apoyo mutuo de los } \\
\text { alumnos de cara a las tareas } \\
\text { produciéndose comunicación } \\
\text { fluida profesor-alumno-alumno. }\end{array}$ \\
\hline $\begin{array}{l}\text { Uso de los } \\
\text { recursos TIC }\end{array}$ & $\begin{array}{l}\text { Las asignaturas involucran el uso de } \\
\text { recursos multimedia sincrónicos y } \\
\text { asincrónicos para realizar sus tareas, sin } \\
\text { embargo, es complicado lograr igual } \\
\text { disposición de todos los alumnos dadas } \\
\text { sus diferentes circunstancias y horarios } \\
\text { de trabajo para utilizar adecuadamente } \\
\text { estos recursos. }\end{array}$ & $\begin{array}{l}\text { La asignatura requiere el uso de } \\
\text { recursos multimedia sincrónicos } \\
\text { y asincrónicos. Además, se les } \\
\text { inicia al uso de otras } \\
\text { herramientas que trascienden a } \\
\text { la mera enseñanza de la lengua } \\
\text { para añadir competencias } \\
\text { informáticas profesionales } \\
\text { transversales. }\end{array}$ \\
\hline $\begin{array}{l}\text { Cumplimiento } \\
\text { del programa }\end{array}$ & $\begin{array}{l}\text { Los syllabus o programas de estudio de } \\
\text { las primeras asignaturas establecen } \\
\text { como objetivo de competencia el nivel } \\
\text { Intermedio-alto en las cuatro destrezas, } \\
\text { de acuerdo con las directrices de ACTFL. } \\
\text { Sin embargo, esta expectativa no se } \\
\text { cumple en la realidad, pues la mayoría de } \\
\text { estudiantes que ingresan al cuarto año } \\
\text { de español, difícilmente han alcanzado el } \\
\text { nivel Intermedio bajo o medio cuando } \\
\text { mucho. }\end{array}$ & $\begin{array}{l}\text { Los alumnos cumplen con el } \\
\text { programa lingüístico } \\
\text { alcanzando una competencia } \\
\text { profesional C1 (en algunos } \\
\text { casos incluso superior a su } \\
\text { competencia de lengua general) } \\
\text { en al menos, lectura y } \\
\text { comprensión auditiva. }\end{array}$ \\
\hline Evaluación & $\begin{array}{l}\text { Limitar la evaluación a las tareas en línea } \\
\text { y ciertos exámenes conlleva una visión } \\
\text { parcial del aprendizaje que aunque es } \\
\text { útil por estar trabajando aunque realizar } \\
\text { el curso online no asegura un } \\
\text { aprendizaje global. }\end{array}$ & $\begin{array}{l}\text { En general se asume, que el tipo } \\
\text { de evaluación asegura un cierto } \\
\text { equilibrio en la competencia } \\
\text { adquirida en cada una de las } \\
\text { cuatro destrezas del lenguaje. } \\
\text { Sin embargo, esta imagen } \\
\text { podría ser superficial ya que la } \\
\text { imagen del profesor muchas } \\
\text { veces (especialmente en la } \\
\text { evaluación colectiva como } \\
\text { presentaciones orales) puede } \\
\text { distorsionar la evolución } \\
\text { individual. }\end{array}$ \\
\hline $\begin{array}{l}\text { Herramientas } \\
\text { de apoyo }\end{array}$ & $\begin{array}{l}\text { Un limitante fuera del control del } \\
\text { instructor es el uso de traductores en } \\
\text { línea, como Google Translate para } \\
\text { elaborar composiciones escritas. El } \\
\text { plagio y la deshonestidad siguen siendo }\end{array}$ & $\begin{array}{l}\text { El trabajo presencial permite un } \\
\text { mayor control en el uso de } \\
\text { herramientas en el centro } \\
\text { docente. Por otro lado, en la } \\
\text { enseñanza presencial los }\end{array}$ \\
\hline
\end{tabular}




\begin{tabular}{|c|c|c|}
\hline & $\begin{array}{l}\text { una de las limitaciones en el } \\
\text { aprovechamiento de estas asignaturas } \\
\text { en línea y una gran frustración para } \\
\text { instructores que sienten no tener el } \\
\text { control ni la misma capacidad de } \\
\text { supervisión que tendrían enseñando } \\
\text { estas asignaturas en el formato } \\
\text { presencial o al menos híbrido }\end{array}$ & $\begin{array}{l}\text { alumnos deben mostrar más } \\
\text { claramente sus capacidades ya } \\
\text { que tienen supervisión y } \\
\text { observación de manera } \\
\text { continuada. }\end{array}$ \\
\hline $\begin{array}{l}\text { Evaluación } \\
\text { externa de la } \\
\text { competencia } \\
\text { profesional }\end{array}$ & $\begin{array}{l}\text { Puede haber una evaluación externa } \\
\text { (generalmente en forma de examen } \\
\text { estandarizado del sistema universitario } \\
\text { del estado para diagnosticar } \\
\text { competencias), y una interna basada en } \\
\text { exámenes escritos y orales, requisitos } \\
\text { todos para completar su certificación } \\
\text { adjunta al ejercicio de su especialización. }\end{array}$ & $\begin{array}{l}\text { La evaluación no es externa por } \\
\text { lo que no tienen un } \\
\text { reconocimiento especial, siendo } \\
\text { esta carencia muy significativa } \\
\text { en su futura vida profesional ya } \\
\text { que para impartir la asignatura } \\
\text { de inglés en enseñanza infantil y } \\
\text { primaria deberán acreditar un } \\
\text { nivel C1 en el CEFR. }\end{array}$ \\
\hline
\end{tabular}

Tabla 5: Comparación de factores limitantes entre UAH y VSU

\section{Conclusiones}

Según se ha expuesto, los programas de VSU y UAH, a pesar de su distinta naturaleza, el primero $100 \%$ en línea y el segundo presencial, comparten varias características tanto en la metodología y materiales utilizados como en el tipo de limitaciones que encuentran tanto alumnos como instructores. En ambos programas, sin embargo, los alumnos cuentan con suficientes oportunidades de aprendizaje ya sea por la disponibilidad de recursos tecnológicos o por la dedicación de los instructores.

Como hemos sugerido en este trabajo la presencialidad tiene un efecto comunicativo dialógico (García Laborda \& García Esteban, 2016) especialmente en la destreza de habla pero en lo referente a lectura, escritura y comprensión auditiva opinamos que no deben aparecer grandes diferencias. Esto se debe al uso de herramientas tecnológicas y didácticas que, sin duda, son comunes en el caso del uso de una plataforma de aprendizaje en ambos casos. También se observan diferencias en el modelo de evaluación y en el uso de herramientas web 2.0 para tareas de escritura y habla en la UAH pero, sin embargo, encontramos muchas semejanzas en el uso del lenguaje y los registros. También entendemos que los objetivos del aprendizaje son más específicos en VSU y centrados en el uso comunicativo en la interacción paciente-médico mientras el uso y aprendizaje de la lengua en la UAH es mucho más amplio. En ambos casos no dudamos que el uso específico de la lengua supere, en algunas ocasiones, incluso el conocimiento 
general de la lengua. En parte esto podría ser por la mayor y más próxima interacción percibida en los alumnos de UAH. Esta mayor interacción se justificaría por la bimodalidad tecnológica- y humana que tienen los alumnos de España. Dicha presencialidad favorece un control más estrecho de los alumnos y saber hasta qué punto consiguen alcanzar un nivel C1 cuando en el grupo de EEUU esto parece no estar asegurado. Quizás, esto sea más fácilmente mensurable debido a la más completa evaluación de la UAH. También se han observado rasgos menores diferenciales como la ética del aprendizaje o las necesidades de evaluaciones externas.

Así pues, mientras en Europa el aprendizaje de lenguas extranjeras para fines específicos se ha convertido en un tipo de aprendizaje estabilizado, los cursos de lenguas para fines específicos en línea como los de VSU proliferan en otras universidades norteamericanas (Lourdes Sánchez-López, 2010), y sus limitaciones han llevado a considerar más asignaturas híbridas (blended learning) ya que las asignaturas en línea no son para todos los estudiantes ni favorecen por igual a los diferentes tipos de aprendices.

Finalmente, algunos de los retos de la enseñanza de programas de lenguas para fines específicos son la calidad y el rigor de la instrucción ya sea presencial o en línea debido a la creciente oferta de asignaturas y titulaciones a nivel internacional. También, los procesos de evaluación de los alumnos tienen que acomodarse a las nuevas tecnologías. Sin duda, la presencialidad reduce la tasa de abandono, pero habrá que observar en un futuro ya cercano si alguna de las dos modalidades tiene un mayor impacto en el aprendizaje de lenguas para fines específicos. En este sentido aunque este trabajo muestra que a pesar de la aparente complejidad de variadas asignaturas y situaciones (Basturkmen, 2010), las características comunes evidencian una gran uniformidad en la enseñanza de lenguas para fines específicos. Quizás en el futuro sea en el área de la mejora de los factores comunes y no de los diferenciadores en donde haya que trabajar buscando criterios comunes de aplicación universal que faciliten la tarea a los profesores internacionalmente.

\section{Sobre los autores}

Jesús García Laborda es Profesor Titular Universidad de Alcalá de Henares (Madrid, España), donde imparte inglés para Educación y Didáctica de la lengua en la Facultad de Educación y Filosofía y Letras. Su investigación se centra en la enseñanza de inglés para fines específicos, evaluación y enseñanza de lenguas asistida por tecnología. Sus numerosas aportaciones incluyen artículos en 
Computers \& Education, British Journal of Educational Technology, Educational Technology \& Society, Language Learning \& Technology o capítulos de libros en editoriales de prestigio como Springer o John Benjamins.

Luis Guillermo Bejarano es Catedrático y Profesor de Honor de lengua española de la Universidad Estatal de Valdosta (Georgia, EEUU) donde imparte asignaturas de didáctica, lingüística y literatura. El Dr. Bejarano recibió el doctorado en literatura hispana de la Universidad de Oklahoma. Además de su interés académico por las letras y los estudios latinoamericanos, ha publicado de su producción original poesía y cuentos en varias revistas literarias de Estados Unidos y América Latina. Asimismo, es notable su trabajo en los proyectos OPENPAU (Universidad de Alcalá) y PLEVALEX (U. Politécnica de Valencia). Durante más de 10 años ha publicado numerosos trabajos junto al co-autor de este artículo en didáctica, evaluación, etc.

\section{LFE Article history}

Paper received: 20 January 2017

Paper received in revised form and accepted for publication: 7 April 2017

\section{Referencias}

Baron, D. (nd). The legendary English-only vote of 1795. Official American English only. Public Broadcasting Service. <http://www.english.illinois.edu/-people-/faculty/debaron/essays/ legend.htm $>[3 / 3 / 2017]$

Bartanusz, S., \& Sulova, L. (2003). Functional analysis of the communication between the young child and his father or mother when reading an illustrated book. European Journal of Psychology of Education, 18(2), 113-134.

Barton, D. \& Hamilton, M. (1998). Local literacies: Reading and writing in one community. London: Routledge.

Basturkmen, H. (2010). Developing Courses in English for Specific Purposes. London, UK: Palgrave Macmillan.

Corder,S. P. (1973). Introducing Applied Linguistics. Harmonds Worth: Penguin.

DeCarlo, M. J. T. (1994). Communicative functions of speech in a monolingual kindergarten. Working Papers in Educational Linguistics, 10(1), 22-31.

Flowerdew, J., \& Peacock, M. (2001). The EAP curriculum: Issues, methods, and challenges. In J. Flowerdew \& M. Peacock (Eds.), Research perspectives on English for academic purposes (pp. 177-194). Cambridge: CUP. 
García Laborda, J. (2011). Revisiting materials for teaching Languages for Specific Purposes. $3 L$ The Southeast Asian Journal of English Language Studies, 17 (1), 102-112.

García Laborda, J. (2013). Reacciones iniciales de los profesores a la preparación de la prueba informatizada de acceso a la universidad. Lenguaje y Textos, 38, 133-140.

García Laborda, J. y García Esteban, S. (2016). Human communicative dialogic practices in content and language-based educational interactions with technology. The Anthropologist, 25(3), 220-228.

García Laborda, J., \& Litzler, M.F. (2015). Current approaches in teaching English for Specific Purposes. Revista Onomázein, 31, 38-51.

García Laborda, J., \& Litzler, M.F. (en prensa). English for Business: Student Responses to Language Learning through Social Networking Tools. ESP Today.

García Laborda, J., Bejarano,L.G., y Simons, M. (2012). ¿Cuánto aprendí en la secundaria? Las actitudes de los estudiantes universitarios de primer año respecto a la relación enseñanza-aprendizaje de su segunda lengua en la escuela secundaria en tres contextos internacionales. Educación XX1, 15(2), 159-184.

Hyland, K. (2004). Genre and Second Language Writing. Ann Arbor: University of Michigan Press.

Howatt, A. P. R. (1984). A History of English Language Teaching. Oxford: Oxford University Press.

Hutchinson, T., \& Waters, A. (1987). English for specific purposes: A learning-centred approach. Cambridge: Cambridge University Press

Ijsewijn, J. (2007). Latin as lingua franca: Renaissance Humanism and translation. En H.. Kittel Kittel, H., Frank, A. P., Greiner, N., Hermans, T.,, Koller, W., Lambert, J., y Paul, F. (Eds.), An international encyclopedia of translation studies. (pp. 1429-1435), New York: De Gruyter.

Johns, A. M. (2002). Genre in the classroom: Multiple perspectives. Mahwah, NJ: Lawrence Erlbaum.

Long, M. (2005). Second language needs analysis. Cambridge, UK: Cambridge University Press.

Sánchez-López, L. (2010). El español para fines específicos: La proliferación de programas creados para satisfacer las necesidades del siglo XXI. Hispania, 93(1), 85-89. 